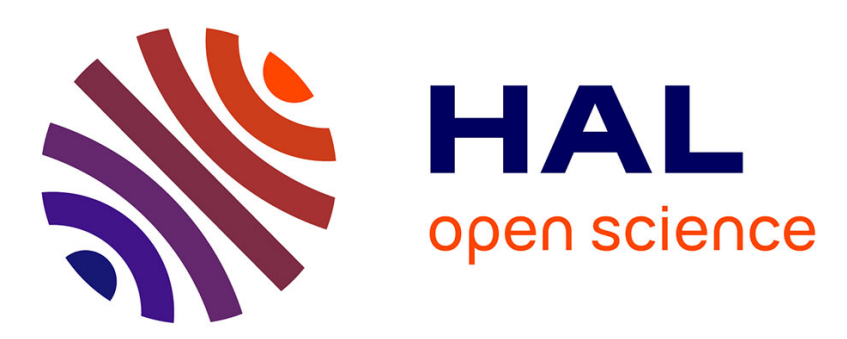

\title{
Gestion de l'eau, grands travaux et pouvoir politique dans le monde mycénien
}

Raphaël Orgeolet

\section{To cite this version:}

Raphaël Orgeolet. Gestion de l'eau, grands travaux et pouvoir politique dans le monde mycénien. Gérer l'eau en Méditerranée au premier millénaire avant J.-C., Presses Universitaires de Provence, 2019, Archéologies méditerranéennes, 9791032002094. hal-03152616

\section{HAL Id: hal-03152616 https://hal.science/hal-03152616}

Submitted on 4 Jan 2022

HAL is a multi-disciplinary open access archive for the deposit and dissemination of scientific research documents, whether they are published or not. The documents may come from teaching and research institutions in France or abroad, or from public or private research centers.
L'archive ouverte pluridisciplinaire HAL, est destinée au dépôt et à la diffusion de documents scientifiques de niveau recherche, publiés ou non, émanant des établissements d'enseignement et de recherche français ou étrangers, des laboratoires publics ou privés. 


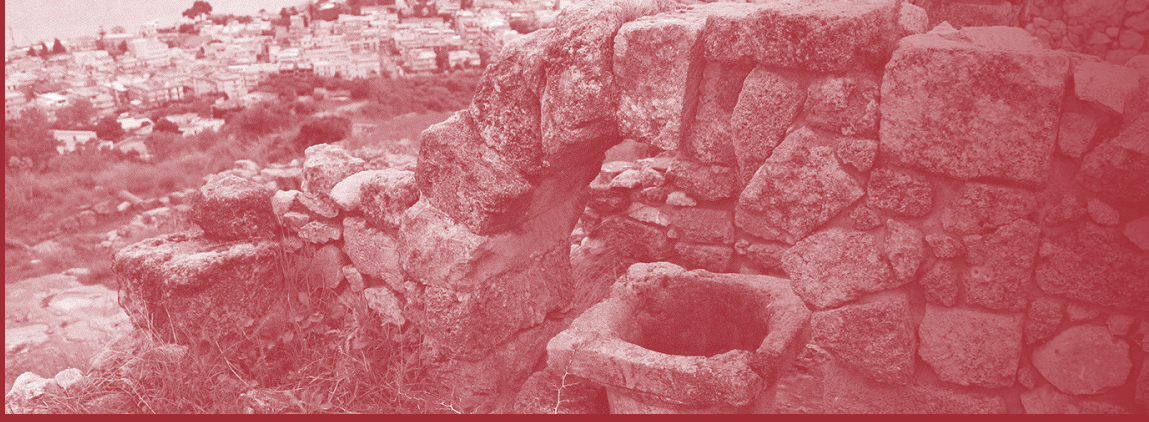

\section{Gérer l'eau \\ en Méditerranée au premier millénaire av.J.-C.} Sous la direction de Sophie Bouffier, Oscar Belvedere et Stefano Vassallo 


\section{ARCHÉOLOGIES MÉDITERRANÉENNES}

collection dirigée par

Marie-Brigitte Carre et François Quantin

Dans la même collection:

Emma MAGLIO, Rhodes: forme urbaine et architecture religieuse (XIVe-XVIII siècles), 180 p., 2016

Colette CASTRUCCI, Le quartier du port de Marseille 1500-1790 : une réalité urbaine restituée, 218 p., 2016.

Prix Félix de Beaujour, Académie des Sciences, Lettres et Arts de Marseille

Catherine DUREUIL-BOURACHAU, Archéologie au présent. Les découvertes de l'archéologie préventive dans les médias, 109 p., 2015

Stéphane BOURDIN, Julien DUBOULOZ et Emmanuelle ROSSO, dir., Peupler et habiter l'Italie et le monde romain, 240 p., 2014 


\title{
Gérer l'eau
}

\section{en Méditerranée \\ au premier millénaire avant J.-C.}

\author{
sous la direction de \\ Sophie Bouffier, Oscar Belvedere \\ et Stefano Vassallo
}


@ Presses Universitaires de Provence

Aix-Marseille Université

29, avenue Robert-Schuman - F - 13621 Aix-en-Provence CEDEX 1 Tél. 33 (0)4 13553191

pup@univ-amu.fr - Catalogue complet sur http://presses-universitaires.univ-amu.fr

DIFFUSION LIBRAIRIES: AFPU DIFFUSION - DISTRIBUTION SODIS 


\title{
Gestion de l'eau, grands travaux et pouvoir politique dans le monde mycénien
}

\author{
Raphaël ORGEOLET \\ Aix Marseille Univ, CNRS, CCJ, \\ Aix-en-Provence, France
}

\begin{abstract}
Résumé - Cet article, au-delà des problématiques techniques qui dominent la production scientifique sur le sujet, aborde les aspects politiques de la maîtrise de l'eau dans le monde mycénien. L'examen des données archéologiques montre un écart très important entre les aménagements domestiques et civils, qui demeurent très modestes, et la démesure d'installations manifestement commanditées et construites par des entités étatiques. Au centre de ces problèmes réside la délimitation des contours de l'État mycénien et de l'étendue de ses prérogatives, que la question de l'eau doit permettre d'aider à définir.
\end{abstract}

\section{Introduction}

La question de l'eau s'est longtemps imposée comme une problématique majeure de la recherche sur les anciennes civilisations du Bassin méditerranéen et de ses prolongements orientaux, qu'il s'agisse de l'Égypte, de la Mésopotamie ou encore de l'ensemble syro-palestinien. La civilisation mycénienne a quant à elle été tenue à l'écart de ce débat, et cela non sans raisons. Les conditions naturelles ne sont bien sûr pas les mêmes en domaine égéen qu'en Égypte ou en Mésopotamie, où la maîtrise de l'eau est souvent une question de survie. Par ailleurs, la précocité des études orientales vis-à-vis de leur benjamine égéenne a constitué un frein à l'histoire comparée: les études mycéniennes étaient encore balbutiantes dans les années 1920 à l'époque où le père Anton Deimel élaborait le concept de cité-temple ${ }^{1}$, et trop occupées à l'exploitation des archives en Linéaire B à peine déchiffré lorsque Karl Wittfogel sortait son Despotisme oriental, au retentissement important et dont les discussions qu'il a initiées jusqu'à sa remise en question rapide ont échappé à l'archéologie égéenne ${ }^{2}$.

Il ne s'agit donc évidemment pas de reprendre ici les termes d'un débat aujourd'hui caduque, posés il y a plusieurs décennies en archéologie orientale. Examiner dans quelle mesure la puissance des institutions étatiques mycéniennes aurait reposé sur la maîtrise de l'eau n'aurait en effet guère de sens. En revanche, l'importance des aménagements hydrauliques dans le monde mycénien, pour les plus ambitieux d'entre eux, ne laisse pas de doutes quant à l'identité de leurs commanditaires: ce sont certainement des réalisations qu'il faut attribuer aux pouvoirs palatiaux.

1 Voir Falkenstein 1954 pour la compilation des travaux d'Anton Deimel (cité par Huot 2005).

2 Wittfogel 1957. Voir également infra p. 7 et n. 26.
Abstract - This paper, beyond the largely addressed technicaloriented issues, takles the political aspect of water management in the Mycenaean world. Archaeological data show a major discrepancy between very modest domestic and ordinary water management related structures, and gigantic ones undoubtly undertaken by the State. Here, one of the key issues is the definition of the Mycenaean State and the realm of its prerogatives, which water management issues could help to address.

Ces aménagements nous adressent aujourd'hui par ailleurs une série de questions dont la première concerne leur nature, et imposent donc d'en établir une typologie. Par delà cette entreprise de mise en ordre, nous devons chercher bien évidemment à déterminer leur fonction. Celle-ci peut se comprendre de manière immédiate : il s'agit de la dimension technique, que nous pouvons identifier comme une réponse tactique à un problème particulier. En second lieu, cette fonction technique - ou tactique - s'insère dans un système plus vaste aux facettes multiples: technique là encore, mais aussi politique et économique. Nous touchons ici à la fonction stratégique de ces installations, dont la détermination permettra de mesurer la place et l'importance de la gestion de l'eau pour les pouvoirs politiques au sein du monde mycénien.

Les ouvrages mycéniens relevant du domaine de la gestion de l'eau et identifiés comme tels, pour impressionnants qu'ils soient, ne constituent probablement qu'une petite partie d'un ensemble typologique certainement beaucoup plus vaste. Dans ce domaine comme dans bien d'autres, les données dont nous disposons proviennent en effet essentiellement de la fin de la période et des sites centraux, et renvoient à la seule sphère palatiale.

Comme établi plus haut, c'est immédiatement à celle-ci que l'on associe spontanément les plus impressionnants des ouvrages d'hydro-ingénierie laissés par les Mycéniens (barrages, canaux et polders), ainsi que les quelques rares vestiges relatifs à l'acheminement et au stockage de l'eau dans les citernes des principales citadelles d'Argolide ou d'Attique. Ces vestiges spectaculaires, qui sont quasiment les seuls à nous être parvenus et dont la fonction est majoritairement le contrôle et la mise à distance d'eaux hostiles, masquent certainement une réalité plus diverse et plus riche. Par ailleurs, le haut degré de technicité et la maîtrise technologique qu'ils ont mobilisés ont focalisé l'attention 
sur leur mise en œuvre, au détriment de leur intégration dans une compréhension globale des problématiques de la maîtrise de l'eau.

Les aménagements hydrauliques mis au jour dans le monde mycénien ne sont donc pas extrêmement nombreux, et il est loin d'être assuré que ceux qui nous restent soient représentatifs de l'ensemble. À cela, deux raisons: en ce qui concerne les agglomérations, ce n'est que relativement récemment (peu ou prou les quinze dernières années) que l'archéologie mycénienne s'est intéressée aux villes, cessant alors de se concentrer sur les seuls palais et forteresses; mais les données issues de ces nouvelles recherches sont encore rarement, sinon pas publiées ${ }^{3}$. L'autre raison tient à ce qu'en milieu rural nous faisons face à la fois à la grande fragilité d'une catégorie potentielle d'aménagements (par exemple les canaux de petites tailles, terminaisons d'un éventuel système d'irrigation) qui se double d'un autre handicap partagé avec les puits et les citernes: leur datation n'est pas toujours assurée.

Malgré ces obstacles, auxquels nous pourrions ajouter le relatif silence des sources écrites, il est utile et nécessaire de revenir sur un sujet dont le traitement demeure déséquilibré dans la littérature spécialisée. En effet, si la « maîtrise de l'eau » (water management) est l'objectif proclamé des analyses qui s'intéressent aux ouvrages du génie hydraulique, c'est bien souvent la seule dimension technique qui est envisagée ${ }^{4}$. Il manque encore une étude qui tenterait d'embrasser l'ensemble des données du problème et à laquelle nous appelons ici. Celle-ci doit intégrer non seulement les paramètres propres aux maîtres d'œuvre, ces États que nous avons évoqués plus haut, mais aussi tous les autres corrélats qui ensemble esquissent les contours d'un système: les conditions naturelles, les vestiges archéologiques ainsi que les usages postulés de la ressource en eau. La proposition qui suit, et qui en raison de l'ampleur du sujet ne pourra être considérée que comme une ébauche, ambitionne la réunion de ces objets.

\section{Le monde mycénien et l'eau: contingences géographiques et climatiques}

Les grands travaux de maîtrise de l'eau par les Mycéniens sont les premières tentatives anthropiques de domestication de la nature d'une telle ampleur et à telle échelle opérées dans cette région de la Méditerranée depuis la fin de l'ère glaciaire. La première des questions devant guider à leur

3 Des travaux sont notamment en cours à Tirynthe (M. Aufschnaiter, cf. http://www.ufg-va.uni-hd.de/forschung/forschungen_tiryns. html, page consultée le 1/03/2016).

4 Par exemple Smith 1995 ou Hagel et Hope Simpson 2006. compréhension renvoie à leur causalité. Ils pourraient en effet aussi bien être le résultat combiné d'une conjoncture favorable - le développement d'entités étatiques disposant à la fois des capacités techniques et politiques pour les réaliser et d'un besoin engendré par la pression maximale exercée par l'homme sur le milieu: l'espace égéen de la fin de l'âge du Bronze, et particulièrement la partie méridionale de la péninsule grecque, est en effet considéré comme un «monde plein », l'ensemble des terres disponibles étant mis en valeur pour satisfaire les besoins d'une région ayant atteint son maximum démographique. La maîtrise de l'eau, dans le cadre d'une démarche visant à conquérir ou à préserver des terres agricoles, trouverait ainsi justification. Une autre explication tendrait à y voir la réponse à un milieu rendu instable, soit de façon conjoncturelle par le changement climatique, soit de façon structurelle par l'action anthropique. Il est entendu que ces deux explications ne sont pas exclusives l'une de l'autre, puisque l'action anthropique est rendue particulièrement vive en raison du maximum démographique ${ }^{5}$.

Au risque de rappeler des évidences, soulignons que la péninsule grecque, avatar méridional de la chaîne balkanique, est caractérisée par un fort relief, tout à la fois omniprésent, contrasté et morcelé ${ }^{6}$. Les plaines y sont rares, qu'il s'agisse des plaines littorales, ou à plus forte raison des plaines intérieures. Le réseau hydrographique, largement structuré par l'importance des systèmes karstiques qui sont notamment particulièrement présents dans le cœur du monde mycénien (nord-est du Péloponnèse et Grèce centrale), est marqué par la multitude des tracés secondaires et la rareté des fleuves puissants et pérennes.

L'empreinte du climat méditerranéen est très sensible sur l'ensemble des territoires qui façonnent le monde mycénien. Celle-ci conduit à l'alternance d'hivers froids qui concentrent la majeure partie des précipitations au début et à la fin de la saison, et d'étés particulièrement chauds et secs. L'ensoleillement et la hausse des températures à la belle saison ne sont pas sans effets sur la ressource hydrique: l'évaporation y est maximale et mène, combinée à l'absence de précipitations, à de fortes sécheresses qui affectent aussi bien les cours d'eau que les sols. Ces intenses contrastes saisonniers se doublent d'une très importante variabilité annuelle, thermique et surtout pluviométrique, puisque les rapports de précipitations peuvent varier d'une année sur l'autre selon un facteur supérieur à 5 en un lieu donné?

5 Suite à la thèse classique de Vita-Finzi (Vita-Finzi 1969), voir notamment Van Andel et al. 1990 pour l'origine anthropique des grands épisodes érosifs de l'Holocène.

6 Voir par exemple FOUACHE 1999: 24-25.

7 Pour une synthèse sur le climat égéen, voir L. Faugères et L. Lespez in Treuil et al. 2008 (respectivement: $1-13$ et 13-30); une approche régionale privilégiant le cœur du monde mycénien est disponible dans Fouache 1999, 25-32. 
Dans ce domaine comme dans celui des paysages, l'espace égéen est marqué par le caractère prépondérant des contingences locales, qui contribuent à en faire une mosaïque de microrégions ${ }^{8}$. De ces quelques remarques découlent deux corollaires principaux: de toute évidence, bien que le monde mycénien soit à cet égard mieux loti que nombre de régions d'Afrique du Nord et du Proche et Moyen Orient où s'épanouissaient à la même époque des civilisations comparables, la question de la ressource en eau y est naturellement aigüe. Par ailleurs, la fragmentation et la compartimentation de l'espace, associées à la très grande variabilité tant spatiale que temporelle des phénomènes climatiques, rend très difficile sinon impossible la restitution de ces problèmes par de simples généralités ${ }^{9}$.

\section{Les Mycéniens et l'eau: ouvrages hydrauliques et logiques d'implantation des sites}

Le premier critère d'évaluation de la relation entre les Mycéniens et l'eau est très certainement celui de la ressource hydrique et de l'implantation des sites d'habitat.

John Bintliff avait établi il y a longtemps que la nature des sols et la proximité de la mer avaient été des critères plus importants que la ressource en eau dans le choix d'implantation des sites mycéniens (Bintliff 1977 : 9). À cela, il faut certainement ajouter, dans un certain nombre de cas au rang desquels celui de Mycènes n'est pas des moindres, des raisons d'ordre stratégique. De toute évidence, un grand nombre des sites de premier rang du nord du Péloponnèse et de Grèce centrale (outre Mycènes, on pense bien sûr à Tirynthe et à Athènes), relève de cette problématique. Le cas des sites méridionaux est différent: le potentiel défensif de Pylos est particulièrement faible, de même que semble l'être celui d'Ayios Vasilios en Laconie, que la fouille inachevée ne permet pas encore d'intégrer à cette réflexion. Pour s'en tenir donc à Pylos, il est également assuré que la zone dans laquelle a été installé le palais n'est pas la région la plus intéressante de Messénie en termes de potentiel agricole et de ressource hydrique: les vallées du Pamisos à l'est et du Steniclaros au nord sont à cet égard bien plus accueillantes. La raison de l'installation du site palatial sur la colline d'Ano Engliano pourrait s'expliquer par l'abri que constitue la baie de Navarin pour les navires, et donc relever d'impératifs tout autres que celui de l'approvisionnement en eau (McDonald et Hope Simpson 1972: 139).

8 Voir en dernier lieu Horden et Purcell 2000 pour ce topos de la géographie historique méditerranéenne, et tout particulièrement égéenne.

9 Les observations ethno-archéologiques effectuées notamment par Hamish Forbes et par Paul Halstead sont très éclairantes à cet égard (Forbes 1982, notamment p. 335; Forbes 2007; Halstead 2014).
Le développement tardif de structures d'adduction d'eau et d'autres aménagements hydrauliques dans les sites de premier rang (à Mycènes et à Pylos notamment), peut témoigner d'un phénomène de pénurie engendré par une pression trop importante sur la ressource. Si l'on extrapole à partir de ces deux cas, on doit avoir à l'esprit que pour la période considérée ici, la période palatiale, les sites centraux mycéniens sont occupés déjà depuis près de quatre siècles au moins et qu'ils sont autant des lieux de mémoire que des positions stratégiques. Le meilleur exemple étant là encore bien sûr celui de Mycènes, dont les monuments funéraires les plus anciens, les cercles A et B, comme la muraille sont des marqueurs spatiaux mais aussi mémoriels. En d'autres termes, les aménagements hydrauliques que nous connaissons renvoient à une période qui n'est pas celle de l'installation, et pour laquelle les besoins et les contraintes étaient nécessairement différents.

\section{Les structures archéologiques de la maîtrise de l'eau}

Les plus connus des ouvrages pour lesquels nous avons quelque certitude, puisqu'il faut ici insister sur la rareté des installations modestes du quotidien, sont aussi les plus spectaculaires et les plus facilement repérables: il s'agit d'un côté des citernes aménagées dans ou à proximité des grandes citadelles (Mycènes, Tirynthe, Acropole d'Athènes), et de l'autre des ouvrages de génie qui visent à endiguer ou à canaliser des cours d'eau, dans le but soit de les détourner, soit de créer des polders ou encore à l'inverse des retenues.

Les paragraphes suivants visent à présenter brièvement la documentation disponible. Les plus importants des travaux de génie hydraulique sont ceux qui visèrent à l'assèchement du lac Kopaïs en Béotie.

\section{Gla et le Kopaiis}

Le projet d'assèchement du lac Kopaïs dans la zone autour de la citadelle mycénienne de Gla, située en Béotie à mi-distance entre Orchomène et Thèbes (fig. 1), constitue en effet certainement l'entreprise de génie mycénienne la plus importante et la plus complexe attestée à ce jour. La décrire ici en détail serait trop long et seul l'essentiel sera évoqué ${ }^{10}$. Dans la partie nord-est du bassin du Kopaïs, qui est aussi la plus basse, ont été mis en évidence tout d'abord dans la seconde moitié du $\mathrm{XIX}^{\mathrm{e}}$ siècle puis après la Seconde Guerre mondiale, différents aménagements permettant la collecte d'une grande partie des eaux du bassin et la poldérisation d'importantes surfaces. Deux types d'ouvrages sont réputés remonter à la période

10 Les principales références concernant Gla et le Kopaïs sont: Iakovidis 2001, Hope Simpson et Hagel 2006 ainsi que les nombreux travaux de J. Knauss, pour la plupart mentionnés dans Knauss 2001. 
mycénienne: des digues et des canaux, tandis que la date du creusement du tunnel de Kephalari à l'extrémité orientale du bassin, qui a été interrompue avant achèvement, est sujette à débats. Un certain nombre d'arguments techniques - aucun canal mycénien ne semble y mener ${ }^{11}$ - et technologiques - le percement d'un tunnel sans le recours à un outillage en fer semble très difficile - doivent nous inciter à nous ranger à l'hypothèse hellénistique (Hope Simpson et Hagel 2006: 204). À la période mycénienne, l'évacuation des canaux semble ne s'être faite que par des exutoires naturels, les katavothrès que l'on retrouve au pied de tout le contrefort karstique oriental du bassin. Les résultats d'une prospection menée dans les années 1980 par John Bintliff et Anthony Snodgrass semblent indiquer que le lac ne s'étendait pas, même au plus fort des hautes eaux de l'hiver, au-delà de $94,5 \mathrm{~m}$ d'altitude (fig. 2) ${ }^{12}$. C'est l'aire autour de Gla qui est la plus basse, dans la partie nord-est du bassin, une zone où le cloisonnement par le relief karstique rendait aussi plus aisées les opérations de construction. Celles-ci ont consisté en l'érection de plusieurs digues propres à l'aménagement de polders, et dont certaines sont encore visibles aujourd'hui (Lane et Aravantinos 2012A: fig. 12). Des tronçons de canaux ont également été relevés, ce qui permet d'aboutir à la restitution la plus généralement admise (fig. 2), avec un long canal principal au nord, long d'environ $25 \mathrm{~km}$ pour ca. $40 \mathrm{~m}$ de large, doublé dans sa partie est d'un second ouvrage jumeau de même largeur. Il était depuis longtemps supposé que cette entreprise devait permettre la mise en culture des terres inondées du bassin du Kopaïs au profit des occupants de l'établissement fortifié mycénien dominant le promontoire de Gla, et c'est désormais chose faite avec les résultats - encore provisoires - du projet AROURA dirigé par Michael F. Lane et Vassilios Aravantinos (Lane et Aravantinos 2012a et 2012b).

\section{Tirynthe}

C'est dans le Péloponnèse, au cœur du monde mycénien, que nous trouvons les autres ouvrages relevant de cette catégorie. L'un des plus importants et plus anciennement connus est l'ensemble formé par le barrage et le canal de dérivation de Tirynthe ${ }^{13}$. À l'époque mycénienne, Tirynthe se trouvait à moins de $500 \mathrm{~m}$ de la côte, contre plus d'1,5 km aujourd'hui. Surtout, l'éperon abritant la citadelle et, plus important encore la ville s'étendant à ses pieds, se trouvaient dans l'emprise du cône alluvial d'un petit cours d'eau aujourd'hui intermittent, mais à la puissance de sédimentation importante au cours de la Protohistoire. Ainsi, on s'est rendu compte tardivement que la citadelle de Tirynthe était environnée d'une ville basse, particulièrement développée sur son flanc méridional: les

11 Néanmoins, on peut aisément comprendre que dans le cas d'un raccordement du réseau de canaux au tunnel, il est évident que le tronçon de raccord aurait été creusé en dernier, après l'achèvement du tunnel.

12 Voir H. Catling, Archaeological Reports 1986-87: 23.

13 Voir Hope-Simpson et Hagel 2006: 182-184 pour l'ensemble des références. niveaux du Bronze Ancien de cette installation se trouvent aujourd'hui à la base d'une stratigraphie de près de $6 \mathrm{~m}$ de haut, très largement constituée d'alluvions (Zangger 1994). Les carottages effectués par Eberhardt Zangger dans les années 1990 sont venus confirmer ce que l'on suspectait depuis les années 1930: la ville basse de Tirynthe a été soumise, depuis le développement de l'agglomération au Bronze Ancien, à de nombreux épisodes diluviaux ayant déposé limons et graviers (Zangger 1993 et 1994). Ces mêmes recherches, combinées aux données issues des fouilles réalisées dans les années 1970 et $1980^{14}$, ont montré que la situation semble avoir été stabilisée à l'HR IIIC et que les travaux à l'origine de ce changement sont à situer à l'HR IIIB2 (dernière phase palatiale) ou au début de l'HR IIIC (phase post-palatiale).

Fig. 1 - Carte figurant les sites mentionnés (R. Orgeolet).

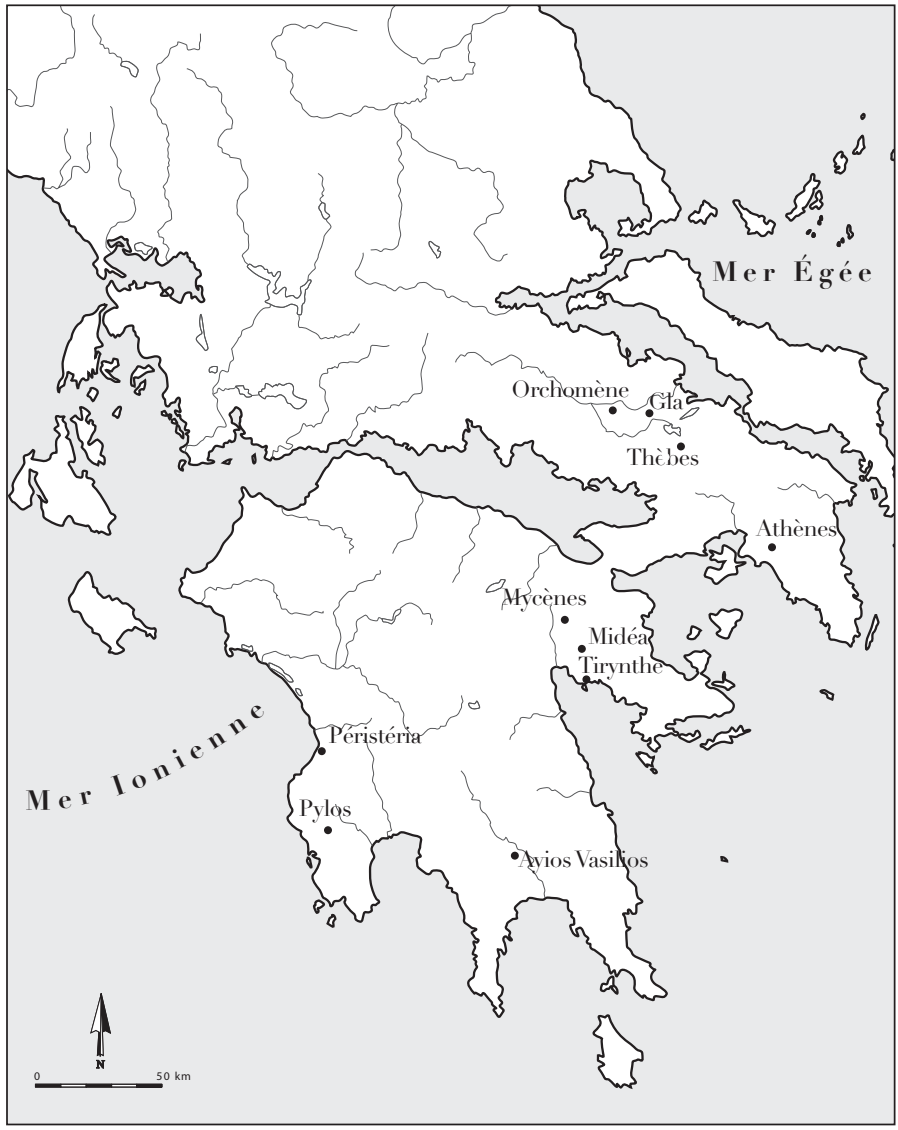

De quelle nature ces derniers étaient-ils? Aux alentours de 1250, un barrage d'une centaine de mètres de longueur et d'une dizaine de hauteur est aménagé en travers du cours d'eau qui jusque-là poursuivait son chemin jusqu'à la mer en passant au nord du Prophitis Ilias et à immédiate proximité de l'éperon de Tirynthe (fig. 3). Cette barrière artificielle a été ménagée avec la terre et les graviers extraits du canal avec lequel elle fonctionne, et renforcée de deux parements

14 Hope Simpson et Hagel 2006: 182 n. 26 pour références. 
Fig. 2 - La région du Kopaïs et les aménagements hydrauliques (d'après Knauss 2004, fig. 12 p. 310).

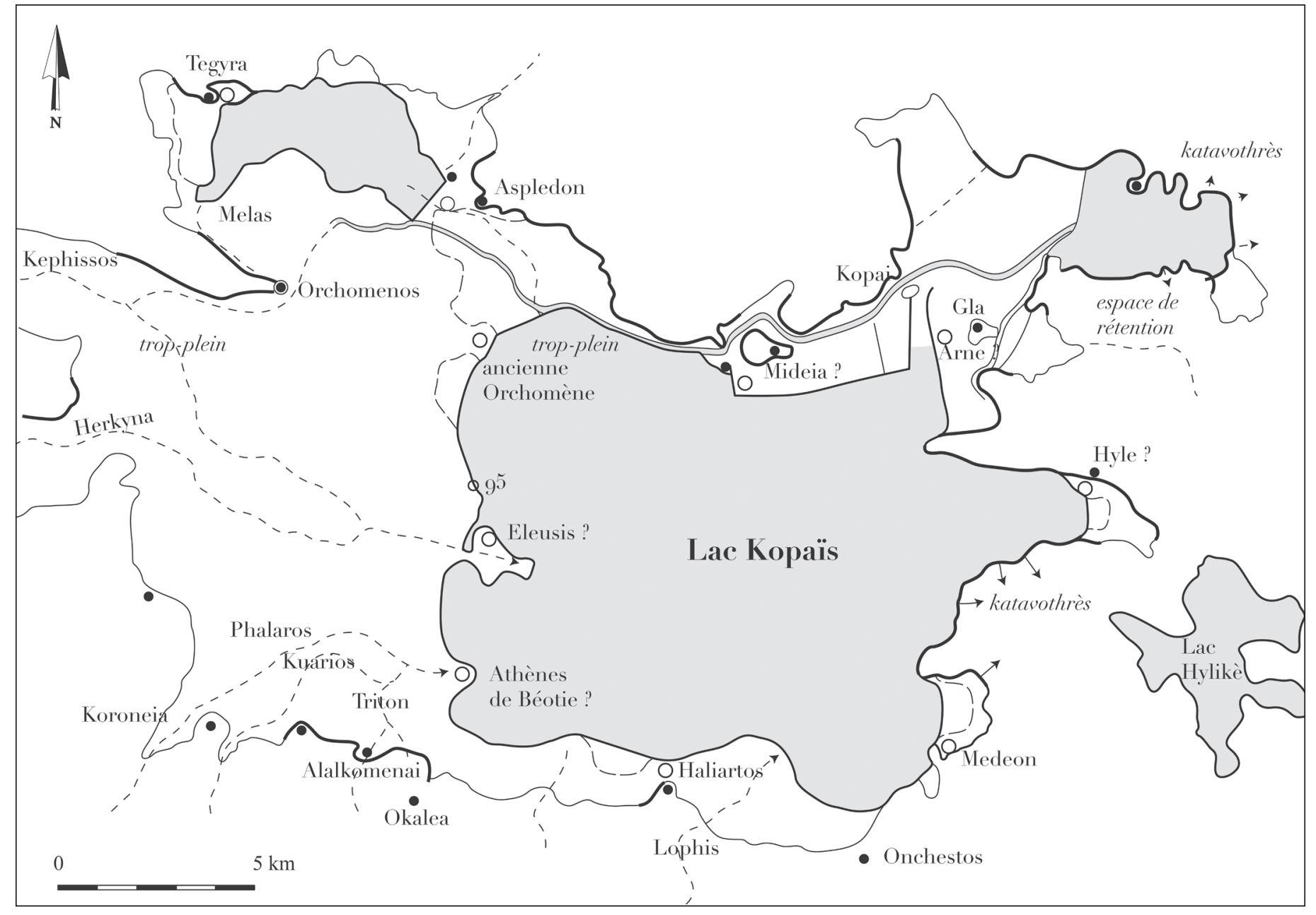

en appareil cyclopéen. Le canal en lui-même est un ouvrage long d'environ $1,5 \mathrm{~km}$, et aujourd'hui large d'environ $40 \mathrm{~m}$ pour une profondeur de $3 \mathrm{~m}$ (Knauss 1996: 82-84). Mais il a manifestement subi des altérations sous l'action de l'érosion, ainsi que des modifications modernes: la physionomie qu'il arborait à la période mycénienne n'est pas totalement connue. Il permettait aux eaux ainsi détournées de rejoindre un autre cours d'eau passant au sud de la citadelle et de son agglomération, qui se sont alors trouvées hors d'atteinte.

\section{Pylos}

Un ouvrage de portée comparable mais de nature et de fonction sensiblement différentes a visé au détournement de la rivière Selas dans la région de Pylos (Zangger et al. 1997). Il s'agit tout d'abord du creusement d'un bassin artificiel dans ce qui était le lit de la rivière Selas au Pleistocène, avant qu'elle ne bifurque vers le sud en raison de l'importance des dépôts alluviaux. Le bassin, qui constitue le port en lui-même, est un rectangle de $400 \times 250 \mathrm{~m}$; il est relié à la mer par un chenal en forme de $\mathrm{S}$ large d'environ $40 \mathrm{~m}$. C'est certainement la partie amont du dispositif qui est la plus intéressante: un canal d'environ $150 \mathrm{~m}$ de long relie le bassin à un lac artificiel, luimême alimenté par la rivière Selas. Cette configuration, bien connue des ports médiévaux, consiste à assurer un courant continu à travers le port en direction de la mer, de façon à interdire l'entrée des eaux marines, extrêmement turbides sur la façade occidentale du Péloponnèse. Le lac artificiel servait de bassin de décantation: les eaux de la rivière, ralenties, y déposaient leurs alluvions qui ne finissaient ainsi pas dans le port. Nous devons tout de même reconnaître quaucune datation n'a pu être assurée pour ce dispositif, l'hypothèse mycénienne n'étant que la plus logique de celles que l'on peut formuler. E. Zangger pense en outre qu'on fermait le canal en hiver, et la Selas s'écoulait tout entière dans son lit habituel, permettant l'irrigation de la plaine et la fertilisation des sols.

\section{Mycènes}

Il n'y a pas à Mycènes d'ouvrage d'une ampleur comparable à ceux du Kopaïs, et de très loin, ni même à ceux de Tirynthe ou de Messénie. La région de Mycènes est en revanche bien connue pour ses routes de la fin de l'âge du Bronze, dont la datation est permise par les murs de soutènement et les 
ponts, bâtis en appareil cyclopéen caractéristique. Pendant longtemps, la construction d'Ayios Georios, qui barre le Chavos à $1 \mathrm{~km}$ en contrebas au sud de la citadelle, avait été assignée à cette catégorie, mais son réexamen par l'hydrologue allemand Jost Knauss a fait douter qu'il se soit effectivement agi d'un pont. Les arguments étaient sa taille (37 $\mathrm{m}$ de long contre 15 pour le pont de Kazarma sur la route d'Épidaure), l'absence de voûte permettant l'écoulement des eaux dans la partie préservée, le caractère dissymétrique de sa construction (la face sud est beaucoup plus robuste que la face nord, et enfin, le fait qu'il ne menait à aucune route (Knauss 1996). Il y a donc de fortes chances pour qu'il ne s'agisse pas d'un pont mais d'un barrage, haut de $4,50 \mathrm{~m}$ et long de $37 \mathrm{~m}$, ménageant une retenue longue de $110 \mathrm{~m}$ et large de $30 \mathrm{~m}$ au maximum; sa capacité est estimée entre 3500 et $4500 \mathrm{~m}^{3}$, pour laquelle on imagine le plus souvent un usage agricole (Hope Simpson et Hagel 2006: 181).

\section{L'eau dans l'habitat mycénien}

\section{L'adduction}

Hormis quelques conduites alimentant essentiellement des dispositifs exceptionnels en lien avec les palais, les agglomérations mycéniennes ne connaissent pas de système d'adduction d'eau. Les ouvrages exceptionnels sont ceux de Mycènes, avec la célèbre citerne orientale de la citadelle ${ }^{15}$, de Tirynthe avec une installation en tous points comparables, c'est-à-dire une source captée, une canalisation et une citerne souterraine ${ }^{16}$, et enfin de Pylos où l'eau d'une source captée est amenée dans la zone du palais au moyen d'une conduite longue d'environ $1 \mathrm{~km}$. C'est là une situation qui n'a rien d'exceptionnel pour des villes de l'Âge du Bronze, et rappelons qu'il en va de même au Moyen-Orient: dans une cité du début du second millénaire comme Mari, dont l'alimentation en eau est assurée par un canal, les bassins et citernes sont remplis par portage (Margueron 2004 : 499).

\section{Le stockage}

Les dispositifs de stockage de l'eau sont rares, et l'on doit tenir les célèbres citernes de Mycènes, Tirynthe et Athènes comme des structures exceptionnelles. En contexte strictement non palatial, seul le site de Malthi en Messénie a livré une citerne (Valmin 1938: 124). Le cas de Midea, bien documenté, ne peut pas être considéré comme relevant de la sphère non palatiale, car il y a tout lieu de penser que le site, sur lequel des documents en Linéaire B ont été retrouvés, était sous la dépendance directe du palais de Mycènes, mais le dispositif

15 Quelques canaux, notamment à proximité des Panagia Houses ont été repérés à Mycènes (cf. Iakovidis 2003: 50, D4-51 et 56, E4-16), ainsi qu’à Thèbes (Demakopoulou et Konsola 197: 44-89).

16 Müller 1930: 61; Biernacka-Lubanska 1980. que l'on y a relevé ${ }^{17}$ semble être de nature plus courante que ceux que l'on retrouve dans les grandes citadelles de Mycènes, Tirynthe et Athènes.

Midea présente le cas d'un site de hauteur, sans accès direct à un cours d'eau ou à une source, et qui a très certainement été choisi pour des raisons stratégiques. Il offre par ailleurs l'exemple le mieux connu, car fouillé et publié récemment, d'un dispositif de gestion de l'eau à l'échelle du site. Le premier élément de celui-ci est constitué par une canalisation collectrice disposée sur le sommet de l'acropole ${ }^{18}$, et qui semble avoir eu pour objectif d'expulser les eaux par-delà la falaise sud. Thea K. Smith, qui a découvert ce dispositif et qui est sur ce point suivie par Gisela Walberg, s'interroge sur son utilité et la possibilité qu'il ait pu servir à irriguer les cultures dans la plaine; cette hypothèse semble bien peu probable, les quantités d'eau en considération étant trop faibles si le dispositif n'est pas complété par un réservoir de grandes dimensions. Or, aucune structure de cette sorte n'a été retrouvée à proximité du sommet de l'acropole, les citernes de Midea se trouvant au contraire et en toute logique dans la partie basse du site.

Ces dernières appartiennent à un ensemble d'installations beaucoup moins énigmatiques, retrouvées à l'opposé du collecteur sommital, à proximité des tronçons nord et ouest de la muraille, sur les terrasses inférieures de l'acropole: il s'agit de deux citernes et de leurs conduites d'alimentation. La Citerne 1 se trouve sous la pièce XVIII (HR IIIB pour son état final). Il s'agit d'une cavité creusée dans la roche d'environ $4,5 \times 3 \times 0,8 \mathrm{~m}$, à laquelle aboutissaient deux conduites provenant de l'amont. Ces dernières étaient constituées de canaux aux parois et au fond plaqués de pierres plates et protégés par des dalles de couverture. On accédait à la citerne depuis la pièce XVII au moyen d'un puisard aux parois recouvertes d'un enduit hydraulique, tandis que la plus grande part de la cavité était recouverte par le sol de la pièce. La seconde citerne, qui se trouve en contrebas près de la muraille, n'a pu être fouillée complètement et ses dimensions exactes ne sont pas connues. Elle était également alimentée par des conduites protégées; aucun élément de couverture n'a été retrouvé. Des tronçons de conduites ont été repérés plus haut sur l'acropole et témoignent d'un dispositif de récupération des eaux de ruissellement à l'échelle du site. L'ensemble de ce dispositif de collecte et de stockage des eaux de ruissellement remonte, d'après l'étude du contenu des citernes et de l'architecture, à la fin de l'Helladique Moyen ou au tout début de la période mycénienne. Des antécédents sont connus à Poliochni, Lerne, Akovitika et Ayia Irini au Bronze Ancien (Walberg 2007: 73).

17 Demakopoulou et al. 1998, Demakopoulou et al. 2000.

18 Smith 1995: 139-141; Walberg 1998: pl. 123d; Walberg 2007: 71. 
Fig. 3 - La région de Tirynthe et les aménagements hydrauliques (d'après Zangger 1994, fig. 13 p. 209).

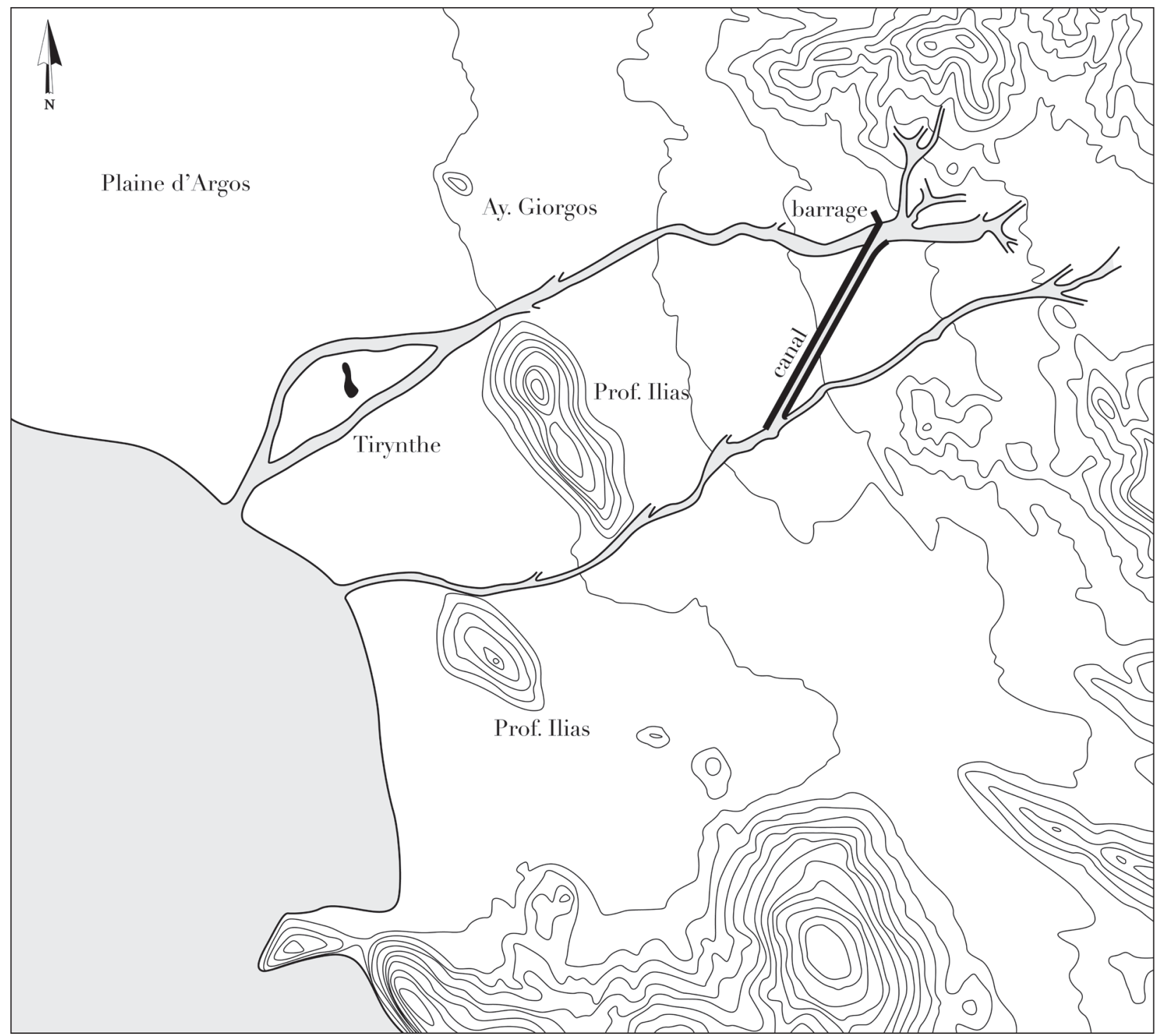

L'exemple de Midea ne doit cependant pas masquer le fait que les dispositifs de stockage de l'eau construits, à l'exclusion donc du stockage en vase, sont rares dans le monde mycénien. Mais cette rareté est loin d'être étonnante, et elle répond à la singularité des structures d'adduction d'eau, que l'on retrouve essentiellement en contexte palatial ou para-palatial comme dans le cas de Midea. Il faut probablement restituer, pour les usages domestiques, des réserves en vases constituées par le recueil des eaux de puits, ainsi qu'une alimentation par portage régulier.

\section{Enjeux pratiques ef techniques}

Avant de revenir en conclusion sur les enjeux politiques de la maîtrise de l'eau, il convient d'esquisser rapidement les domaines dans lesquels ses usages sont les plus importants.

\section{Les usages domestiques de l'eau}

La consommation domestique est bien sûr la première préoccupation pour qui se soucie de l'alimentation en eau d'une agglomération. Nous avons pu constater la rareté des systèmes d'adduction et de stockage de l'eau à grande échelle dans les sites d'habitat mycéniens, et souligner qu'ils étaient l'apanage des palais ${ }^{19}$. L'accès à l'eau devait donc dépendre de la proximité des sources, ou encore de la présence de puits: pour l'agglomération de Mycènes, la source captée bénéficie aux occupants de la citadelle, mais ceux de la ville basse pouvaient compter sur l'existence d'au moins deux puits. Sur le plan domestique, l'usage de l'eau comprend aussi bien la consommation directe que la préparation des repas et, il faut l'imaginer, le nettoyage et la toilette. Des «baignoires", que l'on doit probablement comprendre comme des bassins,

19 Cf. supra p. 18. 
sont ainsi attestées dans toutes les maisons de l'agglomération mycénienne de Iolkos, qui sont également pourvues de canaux d'évacuation des eaux usées (Adrymi-Sismani 2004). Un réseau d'évacuation particulièrement dense et structuré est également attesté à Tirynthe ${ }^{20}$, et il est fait mention de canaux d'évacuation à Iklaina à l'HR III A2 ${ }^{21}$.

\section{La production artisanale}

À la consommation domestique s'ajoutent les besoins des activités artisanales. L'une de celles qui ont laissé le plus de traces, ne serait-ce que par l'importance et la nature de sa production, est l'industrie céramique. On le sait, celle-ci réclame en outre de grandes quantités d'eau pour la préparation de l'argile (Wilson 2000). Les ateliers de potiers sont en revanche peu documentés pour l'époque mycénienne. On en connaît un à Berbati à l'HR II puis à l'HR IIIB, à propos duquel Ann-Louise Schallin pose la question de l'alimentation en eau, absente sur le sommet de cette colline d'Argolide (Schallin 1997: 83). Outre un exemple connu anciennement à Thèbes ${ }^{22}$, des ateliers ont en outre été retrouvés récemment à Tirynthe ${ }^{23}$ ainsi qu'à Mycènes, dans la partie sud-est de la ville basse (Ergon 2008: 35-45). C'est probablement à Mycènes que l'alimentation de l'atelier en eau fut la moins problématique, celui-ci étant situé à proximité du Chavos. Ce même cours d'eau, on l'a vu, a été aménagé de telle sorte à former une retenue. Celle-ci a pu servir à l'alimentation des cultures maraichères (voir infra), mais aussi à d'autres activités artisanales grandes consommatrices en ce domaine: la tannerie et le rouissage du lin (Wilson 2000), deux activités bien attestées à l'époque mycénienne par les tablettes, ont pu bénéficier de cette installation. Notons enfin que la construction des maisons, mais aussi de la plus grande partie des édifices édilitaires (fortifications et tombes monumentales exceptées), fait appel à de grandes quantités de terre crue, dont la préparation nécessite aussi grandes quantités d'eau ${ }^{24}$.

\section{L'agriculture}

L'agriculture est enfin une activité potentiellement grande consommatrice d'eau. Les débats relatifs à la nécessité de pratiquer une agriculture irriguée en Grèce septentrionale à l'époque mycénienne sont anciens, et ne se sont jamais

20 Cf. supra n. 3.

21 http://www.chronique.efa.gr/index.php/fiches/voir/891/

22 Voir Michaelidis 1993: 20 pour les références à la fouille de Keramopoulos en 1911 et les suivantes.

23 http://www.chronique.efa.gr/index.php/fiches/voir/297/

24 Pour la confection des briques, qui est la technique de construction la plus répandue à l'époque mycénienne, il faut compter un volume d'eau pour trois volumes de terre. Pour l'habitat, on peut se faire une idée avec les données suivantes: un mur de maison de $50 \mathrm{~cm}$ d'épaisseur et d'une hauteur de 2,50 $\mathrm{m}$ requerra environ 300 litres d'eau par mètre linéaire (voir ORGEOLET 2008: 44 et Daune-Le Brun 2001). réellement éteints ${ }^{25}$ alors que la nécessité même d'une telle pratique peut être objectivement mise en doute. En effet, il est couramment admis que la limite en deçà de laquelle il n'est plus possible de pratiquer une agriculture sèche correspond à l'isohyète des $200 \mathrm{~mm}$ de précipitations moyennes annuelles, alors que même dans l'une des régions les plus arides du Péloponnèse, le sud de l'Argolide, la moyenne annuelle des précipitations avoisine $500 \mathrm{~mm}^{26}$. À cela on doit ajouter le fait qu'aucune des espèces dont la culture par les Mycéniens est avérée, que ce soit par la découverte de macrorestes végétaux ou par le biais des tablettes inscrites en Linéaire B, ne nécessite absolument d'être irriguée. Il a longtemps été admis que le lin, dont la culture intensive est notamment attestée dans les tablettes pyliennes, nécessitait des sols fertiles et bien irrigués (Chadwick 1963:129). Cette affirmation, notamment en ce qui concerne l'irrigation, a été répétée et amplifiée à plusieurs reprises ${ }^{27}$, pour servir finalement d'argument à l'identification du site mycénien de Nichoria (Messénie) au Ti-mi-to-a-ke-e des tablettes de Pylos. Ce toponyme est en effet associé à la production de lin, et la prospection réalisée par la mission américaine en Messénie a montré que les terres disponibles autour de Nichoria étaient parmi les rares de la région à combiner les critères de fertilité et du potentiel d'irrigation (Shelmerdine 2008: 144). Il n'est pas question de remettre ici en cause l'identification du site archéologique de Nichoria au toponyme mycénien, d'autant que l'industrie du lin nécessite d'énormes quantités d'eau pour le procédé du rouissage. En revanche, la culture du lin ne peut plus être alléguée pour évoquer le recours à une l'irrigation artificielle par l'agriculture mycénienne, les ouvrages d'agronomie comme les témoignages de cultivateurs modernes attestant en effet les capacités de cette plante à se satisfaire de sols pauvres, voire à préférer un environnement $\sec ^{28}$.

Ces débats sont en partie orientés par la perception moderne que nous avons de ces paysages karstiques qui souffrent aujourd'hui du pompage excessif de la nappe souterraine, et il est dangereux de vouloir, comme l'ont fait certains, trop rapprocher sur ce plan l'espace mycénien de la Grèce contemporaine ${ }^{29}$. Ainsi, même si Péloponnèse et

25 Seymour (1908) est le premier qui semble avoir abordé cette question, reprise et développée par la suite (McDonald \& Rapp 1972: 7 \& 14 et suivantes, VAN WERSCH 1972: 184).

26 Voir par exemple Jameson et al. 1994:157.

27 Ventris \& Chadwick 1973: 471; Foster 1981: 76.

28 Voir Biologie du lin 1994 pour un point de vue agronomique moderne; la consultation d'un ouvrage d'agronomie antérieur à la révolution agricole moderne est également édifiante (Bradbury 1920 : 23). Enfin, Pline l'Ancien écrivait déjà que le lin poussait dans des sols sablonneux (Histoire Naturelle XIX, II, 1), ce qui est bien sûr contradictoire avec la nécessité d'une irrigation importante.

29 Comme par exemple W.A. McDonald et R. Hope Simpson: « [...] we here record our belief that in the Late Bronze Age irrigation may well have been used as intensively as nowadays " (McDonald et Hope Simpson 1972: 128). 
Grèce centrale se trouvent résolument dans le domaine de l'agriculture sèche, l'Argolide qui représente l'un des cœurs du monde mycénien, connaît par moment des épisodes de sécheresse qui la conduisent à la limite de la semi-aridité.

Qu'en est-il de l'olivier, une autre plante largement attestée dans la comptabilité mycénienne et assurément l'une des bases de l'économie agricole palatiale? Lynn Foxhall rappelle que les arbres fruitiers, dont l'olivier, ne sont pas élevés à partir de leurs graines, mais plutôt au moyen de greffes, bouturages et marcottage (Foxhall 2007: 97-102). Mais à lire Théophraste, les Grecs anciens pratiquaient avant tout le plantage par souchet pour l'élevage des oliviers, c'est-à-dire par le repiquage de rejetons provenant d'un fragment de souche ancienne ${ }^{30}$. Selon L. Foxhall, contrairement au bouturage, cette technique ne demande pas une abondante irrigation dans les premières années du plant. Là encore, à la condition bien sûr que les techniques des Mycéniens aient été similaires à celles de leurs successeurs et de reconnaître que ce n'est pas certain, nous pouvons conclure que la présence d'une eau abondante, si besoin amenée artificiellement au pied des cultures, n'était pas nécessaire.

Il est cependant à peu près certain que des cultures ont pu être favorisées par l'irrigation: rappelons ici le barrage du Chavos à Mycènes et le canal de dérivation de la Selas dans la région de Pylos, qui ont pu avoir des fins agricoles (voir supra). Il est en outre difficile d'imaginer que les digues et les canaux de Gla n'aient eu pour seul objectif que la formation de polders. Dans ce cas, l'existence d'un réseau de petits canaux venant irriguer les très nombreuses parcelles gagnées sur le lac et les marais, aujourd'hui disparu, est probable. Tout aussi probable est le fait que de l'eau a certainement été artificiellement conduite pour alimenter des potagers et des cultures maraîchères: le barrage du Chavos en bordure méridionale de l'agglomération de Mycènes, même si cela demeure une hypothèse, est le premier exemple venant instantanément à l'esprit.

D'autres aspects des activités agricoles sont dépendants de la ressource en eau: l'élevage, tout comme les cultures, réclame de grandes quantités d'eau. Les tablettes de Pylos comptabilisent environ 10000 ovins, 2000 chèvres, 1000 cochons et une vingtaine de bœufs, soit autant de têtes à abreuver quotidiennement ${ }^{31}$. On peut aussi songer, même si c'est plus anecdotique, au ravitaillement nécessaire des bêtes de somme au cours de leur travail.

30 Histoire des plantes, cité par FoxHALL 2007.

31 Ce fait est notamment rappelé par H. Forbes, qui mentionne l'existence des loutses, ces grands réservoirs aménagés dans les champs du nord de l'Argolide jusque dans les années 1970, destinés notamment à l'abreuvement des troupeaux afin d'éviter une transhumance vers les côtes où se situent les résurgences d'eau (Forbes 1982:34-35 et 121-122).
Il apparaît ainsi que le panorama, rapidement esquissé dans les lignes qui précèdent au sujet de l'irrigation des cultures au cours de la Protohistoire, diffère grandement de celui que nous observons aujourd'hui. De nos jours, les activités agricoles sont en Grèce en effet très largement dépendantes de l'exploitation intensive des ressources en eau et d'un recours massif à l'irrigation. Ce fait, en outre d'avoir par la pression exponentielle exercée sur les réserves, dramatiquement asséché de nombreux cours d'eau et par là même altéré notre appréhension des paysages, présente un tableau certainement très différent de celui qui valait pour la Protohistoire et l'Antiquité. Ainsi, même dans le cas de Gla où la préoccupation principale des bâtisseurs semble avoir été le drainage et l'augmentation des terres agricoles, les grands travaux de génie hydraulique n'avaient pas pour objectif premier l'irrigation des cultures à grande échelle. Cette dernière n'a pu avoir lieu que de manière incidente et secondaire.

\section{Conclusion \\ L'État mycénien et les grands travaux de génie hydraulique: enjeux politiques}

Ce que nous pouvons qualifier de grands travaux de génie hydraulique, catégorie qui accueille aussi bien ceux du Kopaïs, de Tirynthe, de Mycènes ou encore de Messénie, ne se définit pas par une fonction commune, mais plutôt par un ensemble de techniques et de savoir-faire identiques, ainsi qu'une inclination comparable pour la démesure. Leur mise en œuvre s'inscrit sans aucun doute dans le périmètre de l'intervention de l'État.

On ne peut en ce domaine s'empêcher de songer aux problématiques et aux débats relatifs à la maîtrise de l'eau au sein des civilisations urbaines et agricoles du Proche et MoyenOrient ancien, initiés par les théories désormais classiques de $\mathrm{K}$. Wittfogel, qui associait le développement de la puissance étatique à la maîtrise de l'eau (Wittfogel 1957). La question n'est pas ici de juger de la pertinence de ces thèses, qui ont été largement nuancées et remises en question depuis ${ }^{32}$, ni bien sûr de les transposer au cas mycénien.

L'État mycénien, s'il ne fait guère de doute qu'il est bien à l'origine des plus importants des travaux d'ingénierie hydraulique mis au jour, est-il le seul commanditaire dans ce domaine? Quelle que soit l'issue de cette question, quelles peuvent être les motivations de cette implication?

32 Les critiques du Despotisme oriental ont été nombreuses (voir Lees 1994 pour un aperçu). On se contentera ici de mentionner celles de R.M. Adams, qui insistent sur l'absence de documentation archéologique en faveur de l'existence d'un système d'irrigation à grande échelle dans la Mésopotamie de l'âge du Bronze (Adams 1966 et 1981). 
Les mycénologues s'accordent à dire que les contours institutionnels de l'État mycénien sont difficiles à cerner : les tablettes en Linéaire B et autres documents administratifs ne sont que des étapes intermédiaires dans la chaîne bureaucratique et sont fondamentalement à usage interne, s'adressant à des fonctionnaires parfaitement au fait de l'institution et ne s'encombrent pas d'indications superflues. Ainsi, la sphère palatiale peut y être appréhendée et mesurée, mais son importance relative vis-à-vis d'une éventuelle sphère privée ou non-étatique est impossible à déterminer.

C'est exclusivement le gigantisme de ces réalisations qui nous permet, selon une hypothèse très raisonnable, d'en attribuer tout au moins la commande à la puissance souveraine. Il en va autrement de la conduite des travaux, pour laquelle plusieurs scénarios sont imaginables: la maîtrise d'ouvrage a pu être directement gérée par le palais, ou alors confiée à une tierce partie. Remarquons à cet égard que parmi les corps de métiers directement au service du palais et dont les activités apparaissent dans les textes, aucun n'apparaît avec certitude en lien avec la maîtrise de l'eau. Des charpentiers apparaissent dans les archives palatiales: c'est notamment le cas à Cnossos et à Pylos. Dans une tablette de Pylos où ces derniers apparaissent en grand nombre, sont également mentionnés des maçons ${ }^{33}$. Une tablette de Cnossos à l'état fragmentaire présente un hapax curieux: ka-rano-ko ${ }^{34}$. L'ensemble dans lequel ce document figure, la série $\mathrm{B}$, liste des groupes de travailleurs masculins. Selon Petar H. Illievski (Ilievski 1979), contra Jean-Pierre Olivier qui y voit un anthroponyme, ce mot pourrait désigner une profession: gageons que la différence importe peu. Il serait le composé, et la démonstration d'Illievski est convaincante, de ka-ra-na

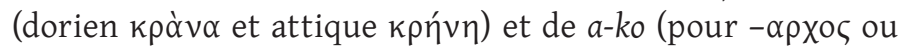
$-\alpha \gamma o \varsigma)$. Si les hypothèses que propose P.H. Illievski justifiant la nécessité d'une telle charge sont insuffisamment étayées, voire aujourd'hui invalidées (voir supra pour ce qui concerne l'agriculture), le principe de l'existence d'un fonctionnaire supervisant la maîtrise des eaux, qu'il s'agisse de leur captation, de leur transport, stockage ou encore répartition, relève du domaine du possible, voire du probable. On pourrait alors s'étonner du caractère unique de cette mention, un hapax qui avait donc conduit J.-P. Olivier à faire de ka-ra$n a-k o$ un anthroponyme. Ce serait néanmoins oublier que les noms de métiers identifiés comme tels par le contexte de l'inscription, mais apparaissant de manière isolée, sont nombreux dans les tablettes mycéniennes.

33 Il s'agit de la tablette Py An 20. Les charpentiers sont désignés sous le

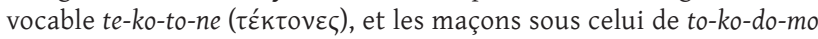

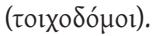

34 Killen et Olivier 1968: 124 : Dv 988 + X 7601 [ + ] B5761.
Est-ce à dire que l'intervention de l'État se limite à ces ouvrages de grande ampleur? Peut-on en déduire que les domaines et les activités concernées par ces ouvrages délimitent en retour le périmètre de l'intervention palatiale?

Nous touchons là au problème des sources et de l'état de notre documentation. Nous devons en effet supposer que les usages de l'eau reposaient également sur tout un pan de structures qui ne nous sont pas parvenues: seules les plus spectaculaires, celles qui ont mobilisé toute la puissance de l'État sont demeurées jusqu'à nous. À l'avenir, des programmes de recherche désormais tournés aussi bien vers les espaces ruraux que vers les centres palatiaux, et déployant des méthodes plus pointues permettant d'affiner notre niveau d'analyse, nous autoriseront peut-être à résoudre ces questions aujourd'hui sans réponse. La compréhension des grands travaux hydrauliques mycéniens, qui le plus souvent s'inscrivent dans une perspective régionale, doit s'effectuer à l'intérieur de ce cadre régional et en relation avec l'ensemble du système formé par le paysage, les acteurs et leurs activités. C'est par exemple le cas, pour évoquer à nouveau les problématiques agricoles, avec la question des terrasses et du modelage des paysages. À Kalamianos sur les rives sud du golfe Saronique en Argolide, à quelques kilomètres de Mycènes, le programme SHARP a récemment révélé l'existence d'un très grand nombre de terrasses agricoles manifestement aménagées au cours d'une entreprise de très grande ampleur, probablement menée par le palais de Mycènes ${ }^{35}$. Un type d'entreprise dont on a tout lieu de penser qu'il englobe à la fois les problématiques de production agricole, de préservation des terroirs par le maintien des terres sur les terrasses, d'approvisionnement en eau, et enfin la mise à distance des eaux dangereuses. Ce n'est que de cette manière que pourront être éclaircies les zones d'ombre qui entravent la compréhension de la maîtrise et de la gestion de l'eau par les Mycéniens. Un domaine qui est encore celui où des ouvrages, que Pausanias aurait en son temps attribué aux Cyclopes, tranchent avec de rares et modestes installations $\mathrm{du}$ quotidien, et où les textes demeurent étonnamment peu diserts.

\section{Bibliographie}

ADAMS 1966: R.M. Adams, The Evolution of Urban Society. Early Mesopotamia and Prehispanic Mexico, Londres, Weidenfeld \& Nicolson, 1966.

ADAMS 1981: R.M. Adams, Heartland of Cities: Surveys of Ancient Settlement and Land Use on the Central Floodplain of the Euphrates, Chicago, The University of Chicago Press, 1981.

ADRYMI-SISMANI 2004: V. Adrymi-Sismani, Le palais de Iolkos et sa destruction, Bulletin de Correspondance Hellénique, vol. 128-129, 2004, p. 154.

35 Kvapil 2012; et Pullen à paraître. 
VAN ANDEL et al. 1990 : T.H. Van Andel, E. Zangger et A. Demitrack, Land Use and Soil Erosion in Prehistoric and Historical Greece, Journal of Field Archaeology, vol. 17, n 4, 1990, p. 379-396.

BINTLIFF 1977 : Mycenaean Geography: Proceedings of the Cambridge Colloquium, September 1976 - Cambridge, The University Library Press, 1977.

BIERNACKA-LUBANSKA 1980: M. Biernacka-Lubanska, The water supply of the Mycenaean citadels and its relations with the Near East, in J. Best et N. De Vries éd., Interaction and Acculturation in the Mediterranean, Vol. I. Proceedings of the Second International Congress of Mediterranean Pre- and Protohistory, Amsterdam, 19-23 November 1980, Amsterdam, B.R. Grüner, 1980, p. 181-189.

BIOLOGIE DU LIN 1994 : La biologie du Linum usitatissimum L. (lin), cahier parallèle aux Critères d'évaluation du risque environnemental associé aux végétaux à caractères nouveaux (BIO1994-10), Agence canadienne d'inspection des aliments, 1994, consulté le 27/04/2016 (bch.cbd. int/database/attachment/?id=2415).

BRADBURY 1920: F. Bradbury, Flax Culture and Preparation, Londres, I. Pitman and Sons, 1920.

CHADWICK 1963: J. Chadwick, The Two Provinces of Pylos, Minos, vol. 7, n² 2, 1963, p. 125-141.

DAUNE-LE BRUN 2001: O. Daune-Le Brun, Les constructions du village néolithique de Khirokitia, Chypre. Reconstitution et expérimentation : premier bilan d'une expérience, in M.C. Belarte, J. Pou, J. Sanmarti et J. Santacana éd., Tècniques constructives d'època ibèrica i experimentació arquitectònica a la Mediterrània: Actes de la 1 Reunió Internacional d'Arqueología de Calafell. Calafell, 20,21 i 22 de gener del 2000, Barcelone, Universidad de Barcelona, 2001, p. 59-73.

DEMAKOPOULOU et KONSOLA 1975 : K. Demakopoulou et D. Konsola,

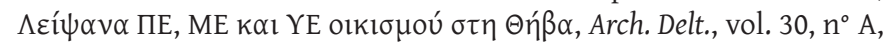
1975, p. 44-89.

DEMAKOPOULOU et al. 1998: K. Demakopoulou, N. Divari-Valakou, P. Åström et G. Walberg, Excavations in Midea 1995-1996, Opuscula Atheninesia, vol. 22-23, 1998, p. 57-90.

DEMAKOPOULOU et al. 2000 : K. Demakopoulou, N. Divari-Valakou, P. Åström et G. Walberg, Work in Midea 1997-1999, Opuscula Atheninesia, vol. 35-52, 2000, p. 57-90.

FALKENSTEIN 1954: A. Falkenstein, La cité-temple sumérienne, Cahiers d'histoire mondiale, vol. I, nº 4, 1954, p. 784-814.

FORBES 1982 : H.A. Forbes, Strategies and Soils: Technology, Production and Environment in the Peninsula of Methana, Greece, Ph.D. dissertation, University of Pennsylvania, Philadelphie, 1982.

FORBES 2007 : H.A. Forbes, Meaning and identity in a Greek Landscape: an Archaeological Ethnography, Cambridge, Cambridge University Press, 2007.

FOSTER 1981 : E.D. Foster, The flax impost at Pylos and Mycenaean landholding, Minos, vol. 17, 1981, p. 67-121.

FOUACHE 1999: E. Fouache, L'alluvionnement historique en Grèce occidentale et au Péloponnèse : géomorphologie, archéologie, histoire, $\mathrm{BCH}$ Supp., n 35, 1999, École française d'Athènes, Paris.

FOXHALL 2007 : L. Foxhall, Olive Cultivation in Ancient Greece: Seeking the Ancient Economy, Oxford, Oxford University Press, 2007.

HALSTEAD 2000 : P. Halstead, Land use in Postglacial Greece, in P. Halstead et Ch. Frederick, Landscape and Land Use in Postglacial Greece, Sheffield, Sheffield Academic Press, 2000, p. 110-128.
HALSTEAD 2014: P. Halstead, Two Oxen Ahead: Pre-Mechanized Farming in the Mediterranean, Hoboken, Wiley-Blackwell, 2014.

HOPE SIMPSON et HAGEL 2006: R. Hope Simpson et D.K. Hagel, Mycenaean fortifications, Highways, Dams and Canals, Sävedalen, Paul Åströms Förlag, 2006.

HORDEN et PURCELL 2000 : P. Horden et N. Purcell, The Corrupting Sea: a Study of Mediterranean History, Oxford, Blackwell, 2000.

HUOT 2005 : J.-L. Huot, Vers l'apparition de l'État en Mésopotamie, Annales HSS, vol. 5, 2005, p. 953-973.

IAKOVIDIS 2001: S. Iakovidis, Gla and the Kopais in the 13th century B.C, Athènes, Archaeological Society at Athens (Library of the Archaeological Society at Athens, no. 221), 2001.

IAKOVIDIS 2003: S. Iakovidis, Archaeological atlas of Mycenae, Athènes, The archaeological society of Athens (Library of the Archaeological Society at Athens, no. 229), 2003.

ILIEVSKI 1979: P. Ilievski, Mycenaean ka-ra-na-ko, SMEA, vol. 20, 1979, p. $161-169$.

JAMESON et al. 1994 : M.H. Jameson, C. Runnels, T.H. Van Andel et M.H. Munn, A Greek Countryside: the Southern Argolid from Prehistory to the Present Day, Stanford, Stanford University Press, 1994.

KILLEN et OLIVIER 1968 : J.T. Killen et J.-P. Olivier, 155 raccords de fragments dans les tablettes de Cnossos, Bulletin de correspondance hellénique, vol. 92, n 1, 1968, p. 115-141.

KNAUSS 1996: J. Knauss, Argolische Studien: alte Strassen-alte Wasserbauten: Talsperre von Mykene, Flussumleitung von Tiryns, Hydra von Lerna, Küstenpass Anigraia, Technische Universität (Bericht / Institut für Wasserbau und Wassermengenwirtschaft und Versuchsanstalt für Wasserbau Oskar v. Miller - Institut in Obernach, Technische Universität München ; 77), Munich, 1996.

KNAUSS 2001: J. Knauss, Späthelladische Wasserbauten : Erkundungen $z u$ wasserwirtschaftlichen Infrastrukturen der mykenischen Welt : Zusammenfassung aller bisherigen Untersuchungsergebnisse, Munich, Technische Universität München, Berichte des Lehrstuhls und der Versuchsanstalt für Wasserbau und Wassermengenwirtschaft der Technischen Universität München, $\mathrm{n}^{\circ}$ 90, 2001.

KVAPIL 2012: L. Kvapil, The Agricultural Terraces of Korphos-Kalamianos: A Case Study of the Dynamic Relationship Between Land Use and SocioPolitical Organization in Prehistoric Greece, Cincinnati, University of Cincinnati, 2012.

LANE et ARAVANTINOS 2012a: M. Lane et V. Aravantinos, Archaeological Reconnaissance of Unexplored Remains of Agriculture (AROURA): Interim Report, 2010-2011 Campaigns, Part 1, Teiresias, vol. 42, n 1A, 2012, p. 214.

LANE et ARAVANTINOS 2012b: M. Lane et V. Aravantinos, Archaeological Reconnaissance of Unexplored Remains of Agriculture (AROURA): Interim Report, 2010-2011 Campaigns, part 2, Teiresias, vol. 42, n 1B, 2012, p. 1526.

LEES 1994: S.H. Lees, Irrigation and society, Journal of Archaeological Research, vol. 2, n 4, 1994, p. 361-378.

MARGUERON 2004 : J.-C. Margueron, Mari : métropole de l'Euphrate au IIIe et au début du IIe millénaire av. J.-C., Paris, Picard, 2004.

MCDONALD et HOPE SIMPSON 1972: W.A. McDonald et R. Hope Simpson, Archaeological exploration, in W.A. McDonald et G.R. Rapp éd., The Minnesota Messenia Expedition. Reconstructing 
a Bronze Age Regional Environment, Minneapolis, University of Minnesota Press, 1972, p. 117-147.

MCDONALD et RAPP 1972 : W.A. McDonald et G.R. Rapp, The Minnesota Messenia Expedition. Reconstructing a Bronze Age Regional Environment, Minneapolis, University of Minnesota Press, 1972.

MICHAELIDIS 1993 : P. Michaelidis, Potters' Workshops in Minoan Crete, SMEA, vol. 32, 1993, p. 739.

MÜLLER 1930: K. Müller, Die Architektur der Burg und des Palastes, Ausburg, B. Filser (Tiryns: Die Ergebnisse der Ausgrabungen des Instituts $\left.n^{\circ} 3\right), 1930$.

ORGEOLET 2008: R. Orgeolet, Espace domestique et architecture en Grèce centrale au Néolithique et au Bronze Ancien, Thèse de doctorat, Montpellier, Université Paul Valéry 2008.

PULLEN à paraître: D.J. Pullen, Agricultural self-sufficiency and Mycenaean Kalamianos on the Saronic Gulf, in D. Garcia, R. Orgeolet, M. Pomadère et J. Zurbach éd., La campagne dans la ville : formes et fonctions des activités agro-pastorales dans la ville pré-classique / Country in the City: Forms and Functions of Agro-Pastoral Activities in Mediterranean Pre-Classical Cities, Oxford, Archaeopress, à paraître.

SCHALLIN 1997: A.-L. Schallin, The Late Bronze Age Potter's Workshop at Mastos in the Berbati Valley, in C. Gillis, C. Risberg et B. Sjöberg éd., Trade and Production in Premonetary Greece: Production and the Craftsman. Proceedings of the 4th and 5th International Workshops, Athens, 1994 and 1995 (Studies in Mediterranean archaeology and literature. Pocket-book $\left.n^{\circ} 143\right)$, Jonsered, P. Åströms Vörlag, 1997, p. 73-88.

SEYMOUR 1908: T.D. Seymour, Life in the Homeric Age, New York, Biblo and Tannen 1963 (1908).

SHELMERDINE 2008: C. Shelmerdine, "Focus. UMME and Nichoria", in J.L. Davis, J. Bennet et S.E. Alcock, Sandy Pylos: an Archaeological History from Nestor to Navarino, Princeton, American School of Classical Studies at Athens, 2008, p. 139-144.

SMITH 1995 : T.K. Smith, Water Management in the Late Bronze Age Argolid, Greece, Cincinnati, PhD. University of Cincinnati, 1995.

TREUIL et al. 2008: R. Treuil, P. Darcque, J.-C. Poursat et G. Touchais, Les civilisations égéennes du Néolithique et de l'Âge du bronze, $2^{\text {de éd., }}$ Paris, Presses universitaires de France (Nouvelle Clio), 2008.

VALMIN 1938: M.N. Valmin, The Swedish Messenia Expedition, Lund, C.W.K. Gleerup, Acta Regiae Societatis Humaniorum Litterarum Lundensis $n^{\circ} 26,1938$.
VENTRIS et CHADWICK 1973: M. Ventris et J. Chadwick, Documents in Mycenaean Greek, Cambridge, Cambridge University Press, 1973.

VITA-FINZI 1969: C. Vita-Finzi, The Mediterranean Valleys: Geological Changes in Historical Times, Cambridge, Cambridge University Press, 1969.

WALBERG 1998: G. Walberg, Excavations on the Acropolis of Midea: Results of the Greek-Swedish Excavations, Stockholm, Svenska Institutet i Athen (Skrifter utgivna av Svenska institutet i Athen, $4^{\circ}$, XLIX), Lund, 1998.

WALBERG 2007: G. Walberg, Midea: The Megaron Complex and Shrine Area. Excavations on the Lower Terraces 1994-1997, INSTAP Academic press (Prehistory Monographs 20), Philadelphie, 2007.

WEIBERG et al. 2016: E. Weiberg, I. Unkel, K. Kouli, K. Holmgren, P. Avramidis, A. Bonnier, F. Dibble, M. Finné, A. Izdebski, C. Katrantsiotis, S.R. Stocker, M. Andwinge, K. Baika, M. Boyd et C. Heymann, The socio-environmental history of the Peloponnese during the Holocene: Towards an integrated understanding of the past, Quaternary Science Reviews, Special Issue: Mediterranean Holocene Climate, Environment and Human Societies, vol. 136, 2016, p. 40-65.

VAN WERSH 1972: H.J. Van Wersh, The Agricultural economy, in W.A. McDonald et G.R. Rapp éd., The Minnesota Messenia Expedition. Reconstructing a Bronze Age Regional Environment, Minneapolis, University of Minnesota Press, 1972, p. 177-187.

WILSON 2000 : A. Wilson, Industrial Uses of Water, in Ö. Wikander (éd.), Handbook of Ancient Water Technology, Boston, Brill, 2000, p. 127-149.

WITTFOGEL 1957 : K.A. Wittfogel, Oriental Despotism: a Comparative Study of Total Power, New Haven, Yale University Press, 1957.

ZANGGER 1993 : E. Zangger, The Geoarchaeology of the Argolid, Berlin, Gebr. Mann, 1993.

ZANGGER 1994 : E. Zangger, Landscape Changes around Tiryns during the Bronze Age, American Journal of Archaeology, vol. 98, n² 2, 1994, p. 189-212.

ZANGGER et al. 1997: E. Zangger, M.E. Timpson, S.B. Yazvenko, F. Kuhnke et J. Knauss, The Pylos Regional Archaeological Project: Part II: Landscape Evolution and Site Preservation, Hesperia: The Journal of the American School of Classical Studies at Athens, vol. 66, $\mathrm{n}^{\circ} 4$, 1997, p. 549-641. 
Table des matières

\section{Gérer l'eau en Méditerranée au premier millénaire avant J.-C.}

Introduction. 5

Sophie BOUFFIER

\section{Comment gérer les eaux en milieu méditerranéen?}

Gestion de l'eau, grands travaux et pouvoir politique dans le monde mycénien

Raphaël ORGEOLET

Springs, sanctuaries and aqueducts in the llissos Valley, Attica,

and the enchanting scenery in Plato's dialogue Phaedrus 25

Eustathios D. CHIOTIS

Investigation of the ancient underground aqueduct in Megara (Greece) 39

Preliminary results

\section{Panagiota AVGERINOU}

Geology and Architectural Conservatism in the Hellenistic Fountains of Corinth and Perachora

Mark LANDON

Qui est l'auteur de l'aqueduc du Galermi? 65

Nouvelles pistes sur un aqueduc plurimillénaire en province de Syracuse (Italie)

Sophie BOUfFIER, Vincent DUMAS, Philippe LENHARDT, Jean-Louis PAILLET, Marcello TURCI

La gestione delle acque a Locri Epizefiri. Criticità e soluzioni, dalle origini alla conquista romana. 87 Diego ELIA 
Installations hydrauliques et usages de l'eau en Ibérie.

\section{Elena H. SÁNCHEZ LÓPEZ}

Les aménagements hydrauliques et la gestion de l'eau

avant la période romaine sur le territoire de l'actuelle Albanie

\section{Lavdosh JAUPAJ}

L'acqua di Mozia: captazione, consumo e significati in ambito civile e religioso.

\section{Lorenzo NIGRO}

\section{Une typologie spécifique: les citernes}

L'approvisionnement en eau des forteresses antiques de la Larisa et de l'Aspis à Argos (Péloponnèse): les trois citernes Vollgraff.

\section{Romaric BARDET}

L'apparition et la diffusion des citernes en Méditerranée phénico-punique...... 169 Un bilan

\section{Iván FUMADÓ ORTEGA}

Gestione e uso dell'acqua ad Himera

\section{Stefano VASSALLO}

Gestione e uso dell'acqua a Solunto: le cisterne.

\section{Giovanni PoLIZZI}

Regenwasser als Ressource in Punischen Siedlungskontexten des zentralen Mittelmeerraumes: Zisternen in Karthago und Pantelleria 


\section{ARCHÉOLOGIES} MÉDITERRANÉENNES

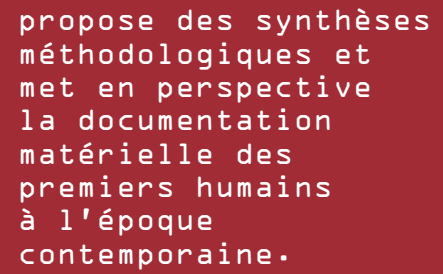

En couverture

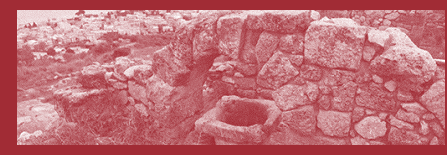

Solunto casa ad atrium tuscanicum. Crédit : Giovanni Polizzi

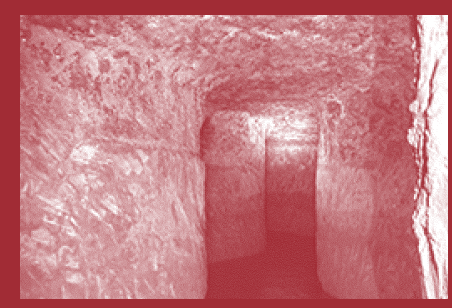

Aqueduc de Galermi (Syracuse), captage du Ciccio. Crédit : Mission Hydrosyra

\section{Gérer l'eau en Méditerranée au premier millénaire av. J.-C.}

À I'heure où la disponibilité et la gestion des ressources en eau de la planète sont au cœur des discussions internationales sur l'avenir de l'humanité, on peut s'interroger sur la manière dont les sociétés antiques ont contrôlé cet enjeu, en particulier en Méditerranée. Dans une tradition historiographique qui exalte la civilisation romaine de l'eau ef lui accorde une place privilégiée, les travaux menés sur les sociétés antérieures n'ont guère retenu l'attention jusqu'à présent. Le réseau HYDR $\Omega$ MED propose d'orienter le regard sur l'adaptation des communautés méditerranéennes, grecques, phéniciennes ou autres, à leurs ressources hydriques. Comment ont-elles géré la pénurie ou la surabondance d'eau? Quelles politiques ont-elles menées pour adapter au mieux leur environnement? Peut-on distinguer politiques publiques et stratégies individuelles? Existe-t-il des savoirs technologiques propres aux différentes cultures et peut-on observer des transferts de compétences d'une communauté à une autre? Le présent volume cherche à répondre à ces questions à travers quatorze articles, avec un focus sur un type d'aménagement hydraulique trop souvent négligé, les citernes, alors qu'il est omniprésent sur les sites méditerranéens. II est le premier d'une série de quatre livres nés de rencontres internationales qui ont réuni à Athènes, Palerme et Aix-en Provence, des archéologues et des historiens soucieux de mettre en lumière les stratégies hydrauliques des sociétés méditerranéennes au jer millénaire avant notre ère.

Sophie Bouffier, ancienne élève de l'École normale supérieure, est professeur d'histoire grecque à I'Université d'Aix-Marseille. Spécialiste de l'expansion grecque en Méditerranée occidentale, elle a consacré une grande partie de ses recherches à l'étude des modes de gestion des ressources hydriques en Sicile et Gaule méridionale.

Oscar Belvedere est professeur de topographie antique à l'Université de Palerme. Spécialiste de l'urbanisme grec et romain et de l'archéologie des paysages, il s'intéresse tout particulièrement aux modes d'occupation territoriale de la Sicile occidentale ainsi qu'aux dynamiques de peuplement gréco-indigène dans cette région.

Stefano Vassallo est directeur de la section archéologique de la Surintendance archéologique de Palerme. Ses recherches portent sur l'expansion grecque en Sicile occidentale et sur les relations entre les populations grecques, indigènes et phénico-puniques. II a mené de nombreuses campagnes de fouilles sur le site d'Himère ainsi que dans I'arrière-pays du littoral tyrrhénien.

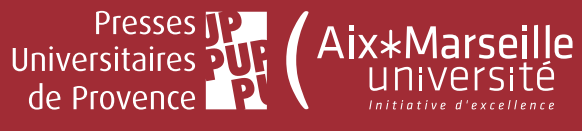

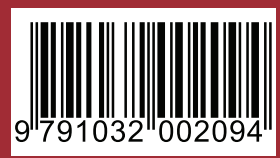

$25 €$ 